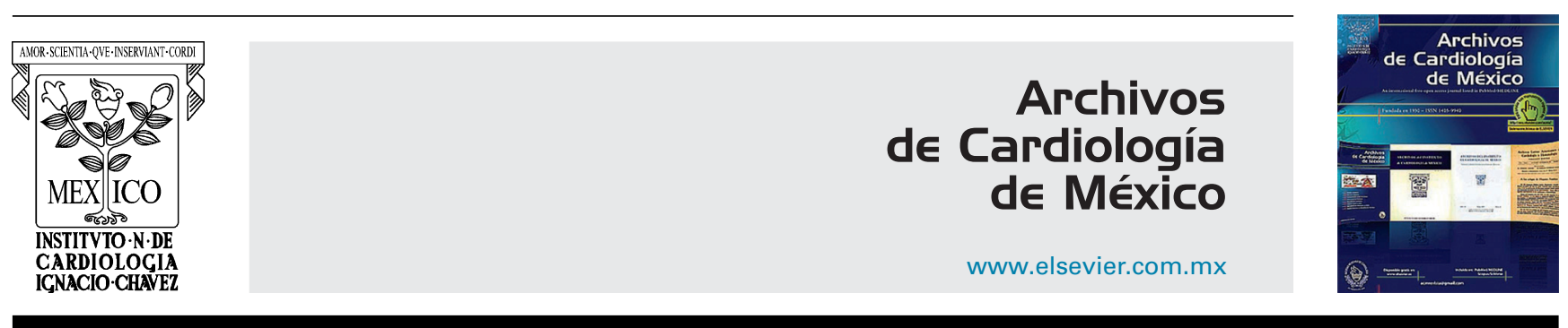

INVESTIGACIÓN CLÍNICA

\title{
Frecuencia, tipo y predictores de complicaciones pleuropulmonares en los primeros treinta días del postoperatorio de pacientes pediátricos intervenidos de cirugía cardiovascular sin apoyo de circulación extracorpórea
}

\author{
Jessica Jacqueline Hernández-López ${ }^{a}$, Alejandro Solano-Gutiérrez ${ }^{\mathrm{b}}$, \\ Flor Teresita Rosas-Aragón ${ }^{c}$, Airam Gabriela Antúnez-Sotoc ${ }^{c}$, Janet Flores-Lujano ${ }^{d}$ \\ y Juan Carlos Nuñez-Enríquez ${ }^{\mathrm{d}, *}$
}

a Unidad Médica de Alta Especialidad, Hospital de Pediatría Dr. Silvestre Frenk Freund, Centro Médico Nacional Siglo XXI, Instituto Mexicano del Seguro Social, Ciudad de México, México

b Unidad Médica de Alta Especialidad, Servicio de Cirugía Cardiovascular, Hospital de Pediatría Dr. Silvestre Frenk Freund, Centro Médico Nacional Siglo XXI, Instituto Mexicano del Seguro Social, Ciudad de México, México

c Facultad de Medicina, Universidad de Sonora, Hermosillo, Sonora, México

d Unidad de Investigación en Epidemiología Clínica, Hospital de Pediatría Dr. Silvestre Frenk Freund, Centro Médico Nacional Siglo XXI, Instituto Mexicano del Seguro Social, Ciudad de México, México

Recibido el 21 de diciembre de 2016; aceptado el 3 de junio de 2017

\author{
PALABRAS CLAVE \\ Complicaciones \\ pleuropulmonares; \\ Cirugía \\ cardiovascular; \\ Cardiopatía \\ congénita; \\ Niños; \\ México
}

\begin{abstract}
Resumen
Objetivo: Determinar la frecuencia, tipo y predictores de complicaciones pleuropulmonares en los primeros 30 días de postoperatorio de pacientes intervenidos de cirugía cardiovascular sin apoyo de circulación extracorpórea.

Métodos: Se realizó un estudio de cohorte retrospectivo durante el periodo comprendido del 1 de enero de 2013 al 31 de diciembre 2014. Incluyó a todos los pacientes portadores de cardiopatías congénitas intervenidos de cirugía cardiaca con abordaje esternal o torácico, sin soporte de circulación extracorpórea con ingreso registrado a Unidad de Cuidados Intensivos del Hospital de Pediatría del Centro Médico Nacional Siglo XXI, IMSS. Se cuantificó la frecuencia de eventos de las complicaciones pleuropulmonares y se realizó un análisis multivariado de regresión logística para identificar los factores de riesgo asociados a complicaciones pleuropulmonares, calculándose odds ratio (OR) e intervalos de confianza al 95\% (IC 95\%).
\end{abstract}

\footnotetext{
* Autor para correspondencia. Unidad de Investigación en Epidemiología Clínica. Hospital de Pediatría Centro Médico Nacional Siglo XXI, Instituto Mexicano del Seguro Social. Av. Cuauhtémoc 330 Col. Doctores, Delegación Cuauhtémoc, Ciudad de México, México. C.P. 06720. Teléfono: +52 5556276900; ext. 22451.

Correo electrónico: jcarlos_nu@hotmail.com (J.C. Nuñez-Enríquez).
} 


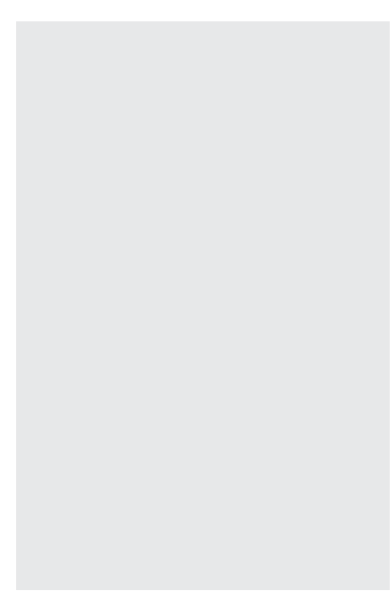

\section{KEYWORDS}

Pleuropulmonary

complications;

Cardiovascular

surgery;

Congenital heart

defects;

Children;

Mexico
Resultados: Se incluyeron un total de 139 pacientes, en los cuales la frecuencia de complicaciones pleuropulmonares fue del $42.4 \%(n=59)$, y los tipos más frecuentes fueron atelectasia (28 eventos), neumonía asociada a ventilador ( 24 eventos), neumotórax ( 20 eventos), pudiéndose encontrar más de una complicación por paciente. Los predictores más significativos de complicaciones pleuropulmonares fueron las cardiopatías congénitas cianógenas $(\mathrm{OR}=3.58$; IC 95\%: 1.10-7.50; $p=0.001)$, el abordaje por toracotomía $(O R=1.46 ; I C 95 \%: 1.18-1.12 ; p=0.008)$ y el evento quirúrgico realizado de urgencia ( $O R=3.46 ;$ IC 95\%: 1.51-7.95; $p=0.002$ ).

Conclusiones: La principal complicación pleuropulmonar fue la atelectasia lo cual concuerda con lo reportado en la literatura internacional. Los pacientes que presenten alguno de los predictores identificados en el presente estudio deben ser monitorizados de manera especial para prevenir, detectar y/o tratar oportunamente las complicaciones pleuropulmonares tras cirugía cardiaca.

(C) 2017 Instituto Nacional de Cardiología Ignacio Chávez. Publicado por Masson Doyma México S.A. Este es un artículo Open Access bajo la licencia CC BY-NC-ND (http://creativecommons. org/licenses/by-nc-nd/4.0/).

Frequency and type of pleuropulmonary complications and their predictors during the first thirty days after cardiopulmonary bypass surgery in children

\begin{abstract}
Objective: To determine the frequency and type of pleuropulmonary complications and their predictors in the first thirty postoperative days of patients undergoing surgery without cardiopulmonary bypass.

Methods: A retrospective cohort study was carried out between January 2013 and December 2014. It included all patients with congenital heart disease who underwent cardiac surgery using a sternal or thoracic approach, without cardiopulmonary bypass with a registered admission to a Neonatal or Paediatric Intensive Care. The frequency of events of pleuropulmonary complications and logistic regression analysis was performed, and the adjusted odds ratio (OR) and confidence intervals at $95 \%(95 \% \mathrm{Cl})$ were calculated.

Results: A total of 139 patients were included. The frequency of pleuropulmonary complications was $42.4 \%(N=59)$, and the most frequent types were atelectasis ( 28 events), ventilatorassociated pneumonia ( 24 events), pneumothorax (20 events), with more than one complication per patient occasionally being found. Significant risk factors were cyanogenic congenital heart disease $(\mathrm{OR}=3.58,95 \% \mathrm{Cl}: 1.10-7.50, P=.001)$, thoracotomy approach $(\mathrm{OR}=1.46,95 \% \mathrm{Cl}: 1.18$ $1.12, P=.008)$, and an emergency surgical event $(\mathrm{OR}=3.46,95 \% \mathrm{Cl}: 1.51-7.95, P=.002)$.

Conclusions: The main pleuropulmonary complication was atelectasis, which is consistent with that reported in the international literature. Patients with any of the predictors identified in the present study should be closely monitored in order to prevent, detect and/or treat pleuropulmonary complications in a timely manner after cardiac surgery.

(c) 2017 Instituto Nacional de Cardiología Ignacio Chávez. Published by Masson Doyma México S.A. This is an open access article under the CC BY-NC-ND license (http://creativecommons. org/licenses/by-nc-nd/4.0/).
\end{abstract}

\section{Introducción}

Las cardiopatías congénitas son un grupo de enfermedades caracterizadas por la presencia de anomalías estructurales del corazón o de los grandes vasos intratorácicos a consecuencia de las alteraciones del desarrollo embrionario ${ }^{1,2}$. En México, se desconoce la prevalencia real de las cardiopatías congénitas por lo que se estima de 8 por cada 1,000 nacidos vivos $^{3-5}$.

De forma muy importante, la innovación en las técnicas de cirugía cardiovascular en niños, la mejora en la protección miocárdica así como del manejo anestésico han permitido que los pacientes pediátricos con cardiopatías congénitas se vean beneficiados cuando son intervenidos quirúrgicamente ya que ha disminuido la tasa de mortalidad postoperatoria. Sin embargo, a medida que se han extendido las indicaciones quirúrgicas, ahora se afronta un incremento significativo en el riesgo de desarrollar complicaciones postoperatorias $s^{6,7}$ y actualmente la probabilidad de muerte después de una complicación es evaluada como una medida de calidad en los pacientes sometidos a cirugía cardiaca ${ }^{8,9}$. Asimismo, se ha visto que en los centros con mayor volumen de pacientes como aquellos de alta especialidad tienen una mayor tasa de muerte en los pacientes que presentan alguna complicación ${ }^{10}$.

En especial, las complicaciones pleuropulmonares postoperatorias son un elemento importante en la incidencia de la morbimortalidad postoperatoria de los pacientes pediátricos 
intervenidos de cirugía cardiaca, ya sea por afectación del propio parénquima pulmonar, alteraciones de presión vascular, la respuesta metabólica al trauma, la manipulación de tejidos o por el tipo de abordaje quirúrgico ${ }^{11}$.

En el cuidado postoperatorio del paciente intervenido de cirugía cardiaca se encuentran una serie de eventos adversos asociados al soporte ventilatorio prolongado $(>72 \mathrm{~h})$, en los cuales se incluyen complicaciones pleuropulmonares, como atelectasias, siendo esta incidencia reportada hasta del $90 \%$ en algunas series de $\operatorname{casos}^{12-14}$; también se encuentran neumonías asociadas a ventilador (NAV), neumotórax, edema pulmonar, parálisis diafragmática, derrames pleurales que se han reportado mayormente en los 2-7 días posteriores a la cirugía ${ }^{15}$, y problemas respiratorios y de la vía aérea como extubaciones fallidas, con una incidencia del $31.9 \%$ y del $20 \%$, si el procedimiento es abierto o cerrado respectivamente ${ }^{16-21}$.

Estudios con grandes series de pacientes sitúan la aparición de complicaciones pleuropulmonares en alrededor del $2.7-4 \%$ y la mortalidad en el $4 \%$, en cirugías electivas, incrementándose los porcentajes hasta el $20 \%$ y el $27 \%$ respectivamente, en procedimientos quirúrgicos de urgencia ${ }^{22}$. A nuestro entendimiento, no existen estudios en nuestro país que hayan evaluado la frecuencia, el tipo de complicaciones pleuropulmonares ni los factores predictores relativos a este desenlace en los primeros 30 días del postoperatorio.

Así, el objetivo del presente estudio fue determinar frecuencia, tipo y predictores de complicaciones pleuropulmonares en los primeros 30 días de postoperatorio de pacientes intervenidos de cirugía cardiovascular, sin apoyo de circulación extracorpórea (CEC).

\section{Pacientes y métodos}

Se realizó un estudio de cohorte retrospectivo. Se incluyeron pacientes de cualquier género y edad en etapa pediátrica portadores de cardiopatía congénita, intervenidos de cirugía cardiovascular con abordaje esternal o torácico, sin CEC y con ingreso registrado a Unidad de Cuidados Intensivos (UCl), ya sea neonatales o pediátricos, en el Hospital de Pediatría «Dr. Silvestre Frenk Freund» del Centro Médico Nacional Siglo XXI, Instituto Mexicano del Seguro Social, en el periodo comprendido del 1 de enero de 2013 al 31 de diciembre 2014. Se excluyó a aquellos pacientes intervenidos de cirugía cardiovascular con CEC y pacientes con mortalidad transoperatoria. Se eliminaron los pacientes que no contaban con expediente completo para el análisis de la evolución posquirúrgica.

Las variables estudiadas fueron: la edad al momento de la cirugía (meses), género, peso al momento de la cirugía $(\mathrm{kg})$, estado nutricional según las gráficas de la OMS 2005 (normal/desnutrición aguda/desnutrición crónica), cromosomopatía (sí/no), tipo de cardiopatía (cianógena/acianógena), cirugía cardiaca previa (sí/no), atelectasia (sí/no), hemotórax (sí/no), neumotórax (sí/no), quilotórax (sí/no), derrame pleural (sí/no), parálisis diafragmática (sí/no), NAV (sí/no), tiempo de ventilación mecánica postoperatoria (días), tiempo quirúrgico (minutos), sangrado transquirúrgico (mililitros), tiempo de estancia en $\mathrm{UCl}$ (días), tiempo de estancia hospitalaria (días), tipo de
Tabla 1 Frecuencia y tipo de complicaciones pleuropulmonares y/o respiratorias de pacientes intervenidos de cirugía cardiovascular sin apoyo de circulación extracorpórea

\begin{tabular}{lll}
\hline $\begin{array}{l}\text { Alguna complicación } \\
\text { pleuropulmonar }\end{array}$ & $\mathrm{n}=139$ & $\%$ \\
\hline Sí & 59 & 42.4 \\
No & 80 & 57.6 \\
Tipo de & Número de eventos \\
$\quad$ complicaciones & & \\
$\quad$ pleuropulmonares & & \\
Atelectasia & 28 & \\
NAV & 24 & \\
Neumotórax & 20 & \\
Parálisis & 7 & \\
$\quad$ diafragmática & 5 & \\
Quilotórax & 5 \\
Derrame pleural & 1 \\
Hemotórax &
\end{tabular}

NAV: neumonía asociada a ventilador.

cirugía (electiva/urgencia) y tipo de abordaje (toracotomía/esternotomía).

Se utilizó el programa estadístico SPSS versión 22, se realizó inicialmente un análisis descriptivo utilizando medidas de tendencia central, dependiendo de la distribución de las variables (media o mediana), y de dispersión (desviación estándar o intervalo) de acuerdo a la distribución de cada variable; posteriormente se llevó a cabo un análisis bivariado para las variables cualitativas y cuantitativas, respectivamente. Se consideró estadísticamente significativo un valor de $p<0.05$.

Posteriormente, se realizó un análisis multivariado de regresión logística, se calcularon odds ratio (OR) e intervalos de confianza al 95\% (IC 95\%), para conocer los factores de riesgo independientes más importantes asociados al desarrollo de complicaciones pleuropulmonares en los primeros 30 días de postoperatorio.

\section{Resultados}

De acuerdo a los registros de procedimientos realizados en quirófano e ingresos registrados a la UCI (Neonatal o Pediátrica) se obtuvieron un total de 354 pacientes intervenidos de cirugía cardiaca sin apoyo de CEC durante el periodo de estudio. Se excluyeron todos aquellos que no contaban con expediente clínico completo $(\mathrm{n}=215)$, quedando un total de 139 pacientes que fueron incluidos en el presente estudio.

Se encontró que, el 42.4\% $(n=59)$ de los pacientes incluidos desarrollaron alguna complicación pleuropulmonar. Se realizó la medición del número de eventos desarrollados durante el periodo de estudio en cada uno de los 59 pacientes que presentaron una complicación pleuropulmonar, observando que la complicación más frecuente fue la atelectasia reportándose 28 eventos, seguida de NAV reportada en 24 pacientes, el neumotórax se observó en 20 pacientes y la parálisis diafragmática en 7 pacientes; de forma individual 5 pacientes presentaron quilotórax y derrame pleural y solo uno presentó hemotórax (tabla 1). 
Tabla 2 Características clínicas del total de pacientes incluidos en el estudio y de acuerdo al desarrollo o no de complicaciones pleuropulmonares en los primeros 30 días de postoperatorio de pacientes intervenidos de cirugía cardiovascular sin apoyo de circulación extracorpórea

\begin{tabular}{|c|c|c|c|c|c|c|}
\hline \multirow[t]{3}{*}{ Variables de estudio } & \multicolumn{4}{|c|}{$\begin{array}{l}\text { Alguna complicación } \\
\text { pleuropulmonar }\end{array}$} & \multirow[t]{3}{*}{ OR (IC 95\%) } & \multirow[t]{3}{*}{$p^{*}$} \\
\hline & \multicolumn{2}{|c|}{ Sí } & \multicolumn{2}{|c|}{ No } & & \\
\hline & $\mathrm{n}$ & $\%$ & $\mathrm{n}$ & $\%$ & & \\
\hline \multicolumn{7}{|l|}{ Tipo de cardiopatía } \\
\hline Cianógena & 29 & 49.2 & 17 & 21.3 & $3.58(1.10-7.50)$ & 0.001 \\
\hline Acianógena & 30 & 50.8 & 63 & 78 & & \\
\hline \multicolumn{7}{|l|}{ Género } \\
\hline Masculino & 33 & 55.9 & 43 & 53.8 & $1.09(0.55-2.14)$ & 0.79 \\
\hline Femenino & 26 & 44.1 & 37 & 46.3 & & \\
\hline \multicolumn{7}{|c|}{ Cirugía cardiaca previa } \\
\hline Presente & 13 & 22 & 6 & 7.5 & $3.48(1.23-9.81)$ & 0.01 \\
\hline Ausente & 46 & 78 & 74 & 92.5 & & \\
\hline \multicolumn{7}{|l|}{ Tipo de abordaje } \\
\hline Toracotomía & 45 & 76.3 & 70 & 87.5 & $1.46(1.18-1.12)$ & 0.008 \\
\hline Esternotomía & 14 & 23.7 & 10 & 12.5 & & \\
\hline \multicolumn{7}{|l|}{ Tipo de cirugía } \\
\hline Urgencia & 21 & 35.6 & 11 & 13.8 & $3.46(1.51-7.95)$ & 0.002 \\
\hline Electiva & 38 & 64.4 & 69 & 86.3 & & \\
\hline \multicolumn{7}{|l|}{ Ingreso a UCIP } \\
\hline Intubado & 47 & 79.7 & 50 & 62.5 & $2.35(1.07-5.12)$ & 0.02 \\
\hline Extubado & 12 & 20.3 & 30 & 37.5 & & \\
\hline \multicolumn{7}{|l|}{ Edad gestacional } \\
\hline Pretérmino & 9 & 50 & 4 & 22.2 & $3.50(1.82-14.84)$ & 0.008 \\
\hline Término & 9 & 50 & 14 & 77.8 & & \\
\hline
\end{tabular}

Tabla 3 Factores de riesgo y morbilidad asociados al desarrollo o no de complicaciones pleuropulmonares en los primeros 30 días de posquirúrgico de pacientes intervenidos de cirugía cardiovascular sin apoyo de circulación extracorpórea

\begin{tabular}{|c|c|c|c|c|c|c|c|}
\hline \multirow[t]{3}{*}{ Variables analizadas } & \multicolumn{2}{|c|}{ Población total analizada } & \multicolumn{4}{|c|}{ Desarrolló complicación pleuropulmonar } & \multirow[t]{3}{*}{$p^{*}$} \\
\hline & \multirow[b]{2}{*}{ Mediana } & \multirow[b]{2}{*}{ Mín-Máx } & \multicolumn{2}{|c|}{$\mathrm{Si}$} & \multicolumn{2}{|r|}{ No } & \\
\hline & & & Mediana & Mín-Máx & Mediana & Mín-Máx & \\
\hline \multicolumn{8}{|l|}{ Factores de riesgo } \\
\hline Edad (meses) & 5 & $0-84$ & 3 & $0-84$ & 5 & $0-84$ & 0.40 \\
\hline Peso $(\mathrm{kg})$ & 4.95 & $0.65-19.4$ & 5.26 & $0.65-19.4$ & 4.92 & $0.69-18$ & 0.74 \\
\hline Duración de la cirugía (min) & 140 & $40-240$ & 150 & $45-240$ & 137.5 & $40-240$ & 0.42 \\
\hline \multicolumn{8}{|l|}{ Parámetros de morbilidad } \\
\hline Duración de VMA (días) & 1 & $0-90$ & 4 & $0-90$ & 1 & $0-20$ & $<0.01$ \\
\hline Días con SEP & 2 & $0-27$ & 3 & $0-27$ & 2 & $0-7$ & $<0.01$ \\
\hline Número de sondas pleurales & 1 & $0-6$ & 1 & $0-6$ & 1 & $0-2$ & $<0.01$ \\
\hline Días en UCIP/UCIN & 4 & $1-119$ & 7 & $1-119$ & 2 & $1-35$ & $<0.01$ \\
\hline Días de ElH & 10 & $3-119$ & 19 & $3-119$ & 7.5 & 3-35 & $<0.01$ \\
\hline
\end{tabular}

EIH: estancia intrahospitalaria; SEP: sonda endopleural; UCIP/UCIN: Unidad de Cuidados Intensivos Pediátricos/Neonatales; VMA: ventilación mecánica asistida.

* U-Mann Whitney. 
Tabla 4 Análisis de regresión logística general para identificar factores de riesgo independientes asociados a complicaciones pleuropulmonares en los primeros 30 días de postoperatorio en los pacientes pediátricos intervenidos de cirugía cardiovascular sin apoyo de circulación extracorpórea, incluyendo el tipo de abordaje

\begin{tabular}{llll}
\hline Variables regresoras & OR & IC 95\% & $\mathrm{P}$ \\
\hline Cardiopatía cianógena & 2.90 & $1.21-6.92$ & 0.01 \\
Tipo de abordaje (toracotomía) & 0.76 & $0.25-2.32$ & 0.64 \\
Tipo de cirugía (urgencia) & 2.35 & $0.92-5.99$ & 0.07 \\
Ingresa intubado a la UCIP & 1.60 & $0.68-3.78$ & 0.27 \\
\hline
\end{tabular}

IC 95\%: intervalos de confianza al 95\%; OR: odds ratio; UCIP: Unidad de Cuidados Intensivos pediátricos.

Modelo ajustado por sexo y edad del paciente al momento de la cirugía.

De los 59 pacientes que desarrollaron una complicación, 26 fueron de sexo femenino (44\%) y 33 del sexo masculino (56\%), con una mediana de edad al momento de la cirugía de 2 y 5 meses para el sexo femenino y masculino respectivamente.

Del total de pacientes que se incluyeron en nuestro estudio, solo se presentó una defunción la cual corresponde al $0.7 \%(n=1)$ de la población, relacionada con neumonía asociada a ventilador. La asociación atelectasia, ventilación mecánica y neumonía asociada a ventilador se presentó en 10 pacientes $(5.8 \%)$ y se asoció con una mayor duración en días de ventilación mecánica $(\mathrm{p}=0.002)$, de estancia en la $\mathrm{UCI}(\mathrm{p}=0.02)$ y de estancia intrahospitalaria $(\mathrm{p}=0.002)$ en comparación con pacientes sin esta tríada; ninguno de ellos falleció en los primeros 30 días del posquirúrgico.

Por otra parte, en el estudio analítico de las diferentes variables estudiadas se observó que los factores de riesgo significativos para presentar un complicación pleuropulmonar en los primeros 30 días de posquirúrgico fueron las cardiopatías de tipo cianógenas $(\mathrm{p}=0.001)$, la presencia de cirugía cardiaca previa $(p=0.01)$, el abordaje por toracotomía $(p=0.008)$, el evento quirúrgico realizado de urgencia $(p=0.002)$, el ingreso a UCl con apoyo de ventilación mecánica asistida $(p=0.02)$ y los pacientes menores de un mes de vida al momento de la cirugía $(p=0.008)$. No se calculó la significación estadística de los diferentes tipos de cardiopatías por la amplia diversidad de la muestra (tabla 2).

Se realizó una recodificación para algunas variables independientes posquirúrgicas, como la duración de ventilación mecánica asistida, días con sonda endopleural, número de sondas pleurales, días en la UCI y los días de estancia intrahospitalaria, encontrando una significación estadística $(p<0.01)$ para presentar mayor morbilidad asociada al desarrollo de complicaciones pleuropulmonares en los primeros 30 días de posquirúrgico (tabla 3 ).

Se realizó un modelo de regresión logística multivariado en donde se incluyeron todas las variables potencialmente asociadas a este desenlace con un valor de $p<0.1$, encontrando que la cardiopatía cianógena $(p=0.01)$ y el tipo de cirugía realizada de forma urgente $(p=0.07)$ representan los factores de riesgo independientes más importantes para desarrollar una complicación pleuropulmonar (tabla 4). De igual forma, al identificar los factores de riesgo específicos por subtipos de complicaciones pleuropulmonares encontramos que el tipo de abordaje por toracotomía confiere un riesgo de 4.56 veces (IC 95\%: $1.32-15.75 ; p=0.01$ ) mayor, para desarrollar NAV. Sin embargo, no se encontraron en el presente estudio factores de riesgo para atelectasia, neumotórax, hemotórax, quilotórax o derrame pleural, posiblemente debido al tamaño de muestra insuficiente.

\section{Discusión}

Dentro de los resultados encontrados, la frecuencia de complicaciones pleuropulmonares reportada fue del $42.4 \%$, valor porcentual elevado respecto a lo encontrado en la literatura nacional e internacional ${ }^{23}$. Aunque la causa probable de esta elevada frecuencia de complicaciones pleuropulmonares pudo deberse al tamaño de la muestra (ya que más de la mitad de los pacientes que se intervinieron de cirugía de tipo cerrado durante el periodo de estudio fueron excluidos por presentar un expediente incompleto para el análisis de su evolución y lo cual consideramos como una pérdida importante de la muestra), por un lado, nos permitió identificar un área de oportunidad importante de mejora para el área de archivo de nuestro hospital; pero, por otro, dado que antes de revisar el expediente clínico no conocíamos cuáles pacientes habían desarrollado algún tipo de complicación pleuropulmonar disminuye la posibilidad de que ocurriera un sesgo en la selección de la muestra y que esto se hubiera relacionado con una frecuencia alta de complicaciones pleuropulmonares. Otro factor pudo ser el tipo de población pediátrica atendida en nuestro hospital (niños con cardiopatías cianógena complejas, con cirugías cardiacas previas y el tiempo de evolución de la cardiopatía), el cual es un hospital de concentración de tercer nivel de atención.

Puntualizamos, de acuerdo a los resultados encontrados y coincidiendo con la literatura, que la atelectasia permaneció como la primera complicación a nivel pleuropulmonar ${ }^{12,14}$, presentándose en 28 de los 59 pacientes que desarrollaron una complicación en nuestro estudio. Duggan et al. reportan como uno de los factores determinantes para su desarrollo el tipo de abordaje quirúrgico ${ }^{20}$; otros autores como Mhanna et al. relacionaron a la cirugía cardiaca con una mayor asociación con atelectasias por el aumento de la permeabilidad capilar y el edema alveolar ${ }^{24}$.

De igual forma las infecciones siguen siendo un factor de complicación prevalente e incluso se relacionan con la mortalidad, registrándose la NAV como la segunda causa de infección nosocomial en la literatura Internacional ${ }^{25}$ y coincidiendo con los datos obtenidos en nuestro estudio observándose como la segunda complicación a nivel pleuropulmonar en nuestra población de estudio; así también la única defunción que se registró estuvo relacionada con esta complicación

La cuestión que se plantea con los resultados obtenidos para el manejo oportuno de estos pacientes es la siguiente: ¿Serán los factores como la neumopatía previa, la inmadurez pulmonar, la traqueobroncomalacia, la cardiopatía, entre otros, los que hacen que el paciente intervenido de cirugía cardiovascular presente una complicación que dificulte la distinción real de su evolución, o el factor determinante para su evolución sea meramente quirúrgico? 
Conseguir que la investigación dé resultados, refleje realmente la actividad realizada y pueda compararse con la de otros grupos es un primer paso que entraña enormes dificultades. Se necesita tener datos fidedignos de los resultados de la actividad de cada grupo quirúrgico (lo que requiere desarrollar sistemas adecuados para la recolección de datos, y establecer criterios uniformes de valoración y clasificación); estos resultados deben ser analizados de manera apropiada, evitando interpretaciones sesgadas, y los datos obtenidos deben ser contrastados con la experiencia general y la de otros grupos. Aplicar la metodología de estimación del riesgo supone conocer qué factores pueden estar relacionados causalmente con los riesgos posibles, cómo determinar su presencia y qué peso atribuir a cada uno a la hora de realizar un pronóstico sobre el riesgo de una intervención.

\section{Conclusiones}

Las complicaciones pleuropulmonares durante los primeros 30 días del posquirúrgico se presentaron en el $42.4 \%$ de la población estudiada, y se asociaron con una mayor estancia en $\mathrm{UCl}$, días de ventilación mecánica y de estancia intrahospitalaria, siendo la atelectasia la principal de ellas, seguida de la neumonía asociada a ventilador. Con el presente estudio se pudieron identificar como subgrupos de alto riesgo de presentar complicaciones pleuropulmonares a aquellos portadores de cardiopatías congénitas cianógenas y aquellos intervenidos de urgencia. Además, que el abordaje por toracotomía se asociaba con un mayor riesgo para el desarrollo de NAV.

\section{Responsabilidades éticas}

Protección de personas y animales. Los autores declaran que para esta investigación no se han realizado experimentos en seres humanos ni en animales.

Confidencialidad de los datos. Los autores declaran que en este artículo no aparecen datos de pacientes.

Derecho a la privacidad y consentimiento informado. Los autores declaran que en este artículo no aparecen datos de pacientes.

\section{Financiación}

No se obtuvo financiamiento para la realización del presente trabajo

\section{Conflicto de intereses}

Los autores declaran no tener ningún conflicto de intereses.

\section{Bibliografía}

1. Baltaxe E, Zarate I, Calderon. Prevalencia de malformaciones cardiacas congénitas en 44,985 nacimientos en Colombia. Arch Cardiol Méx. 2006;76:263-8.
2. Durán P. Cardiopatías congénitas más frecuentes. Pediatr Integral. 2008;XII:807-18.

3. Martinez O, Romero C, Alzina A. Incidencia de las cardiopatias congénitas en Navarra (1989-1998). Rev Esp Cardiol. 2005;58:1428-38.

4. Instituto Nacional de Estadistica y Geografia (INEGI) [consultado 7 Jul 2015]. Disponible en: http://www.inegi.org.mx/ sistemas/temas/default.aspx

5. Calderón J, Cervantes JL, Curi PJ, et al. Problemática de las cardiopatías congénitas en México. Propuesta de regionalización. Arch Cardiol Mex. 2010;80:133-40.

6. Jacobs JP, Jacobs ML, Mavroudis C, et al. Executive summary: The Society of Toracic Surgeons Congenital Heart Surgery database Fifth Harvest 2002-2004. The Society of Torácic Surgeons (STS) and Duke Clinical Research Institute (DCRI). North Carolina, USA: Duke University Medical Centre, Durham; 2005.

7. Jacobs JP, Jacobs ML, Maruszewski B, et al. Current status of the European Association for Cardio-Thoracic Surgery and The Society of Thoracic Surgeon Congenital Heart Surgery Database. Ann Thorac Surg. 2011;80:2278-83.

8. Ghaferi AA, Birkmeyer JD, Dimick JB. Variation in hospital mortality associated with inpatient surgery. New Engl J Med. 2009;361:1368-75.

9. Ghaferi AA, Birkmeyer JD, Dimick JB. Complications, failure to rescue, and mortality with major inpatient surgery in medicare patients. Ann Surg. 2009;250:1029-34.

10. Pasquali SK, Li JS, Burstein DS, et al. The association of center volume with mortality and complications in pediatric heart surgery. Pediatrics. 2012;129:370-6.

11. Gouin F, Guillen JC. Complications respiratoires postopératoires. Encycl Méd Chir (Elvesier, Paris), Anesthesie-Réanimation. 1996:10p, 36-422-A-10.

12. Hazinski TA. Atelectasis. En: Cherniak B, Kendig E, editores. Kendig's disorders of the respiratory tract in children. $6 .^{\mathrm{a}}$ ed. Philadelphia: W.B. Saunders Company; 1998. p. 634-41.

13. Magnusson L, Spahn DR. New concepts of atelectasis during general anaesthesia. Br J Anaesth. 2003;91:61-72.

14. Duggan M, Kavanagh BP. Pulmonary atelectasis: A pathogenic perioperative entity. Anesthesiology. 2005;102:838-54.

15. Wimmwer GG, Yoseeff HM, Rinner T, et al. Effects ofinternal thoracic artery preparation on blood loss, lung function and pain. Ann Thorac Surg. 1999;67:1078-82.

16. Fischer JE, Allen P, Fanconi S. Delay of extubation in neonates and children after cardiac surgery: Impact of ventilatorassociated pneumonia. Intensive Care Med. 2000;26:942-9.

17. Ip P, Chiu CS, Cheung YF. Risk factors prolonging ventilation in young children after cardiac surgery: impact of noninfectious pulmonary complications. Pediatr Crit Care Med. 2002;3:269-74

18. Tulla H, Takala J, Alhava E, et al. Respiratory changes after open-heart surgery. Intensive Care Med. 1991;17:365-9.

19. Cernadas CM. Distribución ventilación/perfusión e insuficiencia respiratoria. En: Dvorkin MA, editor. Bases fisiológicas de la práctica médica. Castellana; 2003. p. 165-76.

20. Hubble CL, Gentile MA, Tripp DS, et al. Deadspace to tidal volume ration predicts successful extubation in infants and children. Crit Care Med. 2000;28:2034-40.

21. Kurachek SC, Newth CJ, Quasney MW, et al. Extubation failure in pediatric intensive care: A multiple-center study of risk factors and outcomes. Crit Care Med. 2003;31:2657-64.

22. Hedenstierna G. Alveolar collapse and closure of airways: Regular effects of anesthesia. Clin Physiol Funct Imaging. 2003;23:123-9.

23. Cooper DS, Jacobs JP, Chai PJ, et al. Pulmonary complications associated with the treatment of patients with congenital cardiac disease: Consensus definitions from the Multi-Societal Database Committee for Pediatric and Congenital Heart Disease. Cardiol Young. 2008;18:215-21. 
24. Mhanna MJ, Zamel YB, Tichy CM, et al. The air leak test around the endotracheal tube, as a predictor of postextubation stridor, is age dependent in children. Crit Care Med. 2002; 30:2639-43.
25. Almuneef M, Memish ZA, Balkhy HH, et al. Ventilator associated pneumonia in a pediatric intensive care unit in Saudi Arabia: a 30-month prospective surveillance. Infect Control Hosp Epidemiol. 2004;25:753-8. 